\title{
De l'Amicale des ex-prisonniers politiques de Silésie à la Fondation Auschwitz : constructions de mémoires en Belgique
}

Van de Vriendenkring van Gewezen Politiek Gevangenen in Silezië tot de

Stichting Auschwitz : bouwen aan de Belgische gedachtenis

Sarah Timperman

\section{(2) OpenEdition}

Journals

\section{Édition électronique}

URL : https://journals.openedition.org/temoigner/6532

DOI : 10.4000/temoigner.6532

ISSN : 2506-6390

\section{Traduction(s) :}

Van de Vriendenkring van Gewezen Politiek Gevangenen in Silezië tot de Stichting Auschwitz: bouwen aan de Belgische gedachtenis - URL : https://journals.openedition.org/temoigner/6579 [nl]

Éditeur :

Éditions du Centre d'études et de documentation Mémoire d'Auschwitz, Éditions Kimé

Édition imprimée

Date de publication : 1 octobre 2017

Pagination : 120-131

ISBN : 978-2-930953-01-4

ISSN : 2031-4183

\section{Référence électronique}

Sarah Timperman, «De l'Amicale des ex-prisonniers politiques de Silésie à la Fondation Auschwitz : constructions de mémoires en Belgique ", Témoigner. Entre histoire et mémoire [En ligne], 125 | 2017, mis en ligne le 24 décembre 2021, consulté le 04 février 2022. URL : http://journals.openedition.org/ temoigner/6532; DOI : https://doi.org/10.4000/temoigner.6532 
De l'Amicale des ex-Prisonniers Politiques de Silésie à la Fondation Auschwitz : constructions de mémoires en Belgique

$\rightarrow$ Sarah Timperman

ès la fin de l'Occupation se pose, en Belgique comme dans les autres ays d'Europe, la question de la mémoire de la Seconde Guerre mondiale. Celle-ci va produire des mémoires concurrentes qui s'excluent mutuellement. La mémoire de l'Occupation en Belgique a d'emblée été monopolisée par les anciens prisonniers politiques qui avaient en charge l'organisation de la transmission du souvenir. Dans les deux premières décennies d'après-guerre, la souffrance des Juifs est absente des commémorations, la mémoire de la guerre est strictement patriotique et combattante. Il faut attendre les années 1960, mais surtout les années 1980 pour quémerge une prise de conscience de l'opinion publique du sens véritable de l'extermination des Juifs. L'amplification du discours négationniste, la montée de l'extrême droite et le regard posé par la communauté juive sur son propre passé amènent progressivement à l'acceptation du caractère unique de la Shoah. Au cours des années 1990, la mémoire du judéocide est au centre de l'attention et Auschwitz devient un des symboles de la Seconde Guerre mondiale. En parallèle, on assiste a contrario au refoulement symbolique des prisonniers politiques. Un demi-siècle après la fin de la Seconde Guerre mondiale, on est passé ainsi d'une mémoire patriotique excluant l'expérience juive à une mémoire de la Seconde Guerre mondiale qui se revendique «des droits de l'homme» dans laquelle le génocide des Juifs est central.

Cet article ${ }^{1}$ se propose d'analyser comment l'Amicale Belge des ex-Prisonnier Politiques de Silésie - devenue en 1976 l'Amicale belge d’Auschwitz et prédécesseur de l'actuelle Fondation Auschwitz - s'inscrit dans cette évolution. Dans le paysage des mémoires éclatées de la Seconde Guerre mondiale, comment une amicale - à la fois «politique » et «d'Auschwitz» - qui se trouve donc au carrefour des deux principales mémoires a-t-elle pu se structurer autour de cette double appartenance? Pour répondre à ces questions, nous nous sommes basés sur les archives de l'Amicale de Silésie conservées à la Fondation Auschwitz et sur les interviews de ses dirigeants réalisées au début des années $1990^{2}$.

CRÉATION DE LAMICALE DES EX-PRISONNIERS POLITIQUES DE SILÉSIE (1946)

Afin de défendre les intérêts des déportés rentrés au pays, de nombreuses amicales de camps voient le jour dans l'immédiat après-guerre. Parmi celles-ci, une amicale d'anciens prisonniers politiques des camps de Silésie (Pologne) dont on trouve la première trace au mois d'octobre $1945^{3}$. Ce groupe de rescapés existe alors de manière informelle et se manifeste afin qu’un écho plus large soit fait au procès de Belsen au cours duquel comparait une quarantaine de SS des camps d'Auschwitz et de Bergen-Belsen. Six mois plus tard, le 31 mars 1946, les membres de cette association se réunissent - à la Vlaamsch huis sur la Grand-Place de Bruxelles - pour

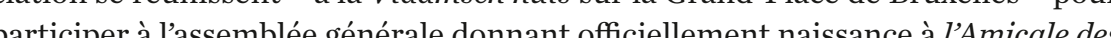

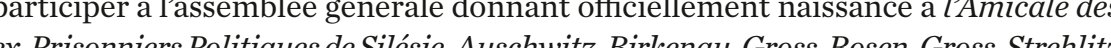
ex-Prison ars Politigestrehlitz et commandos extên ieurs. Lassociation nouvellement creée défhit son programme d’action comme suit : participer à la répression des crimes de guerre et à la chasse

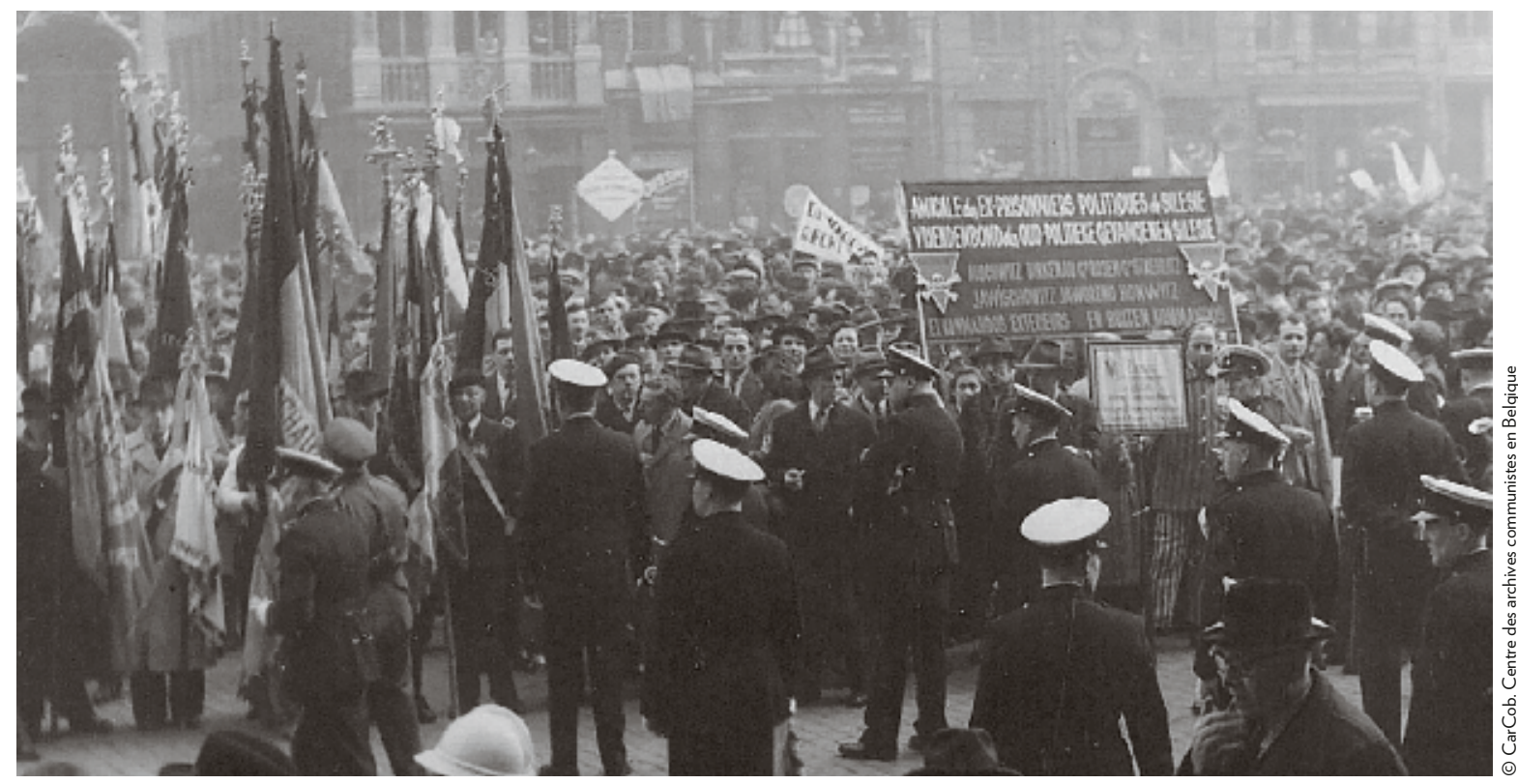




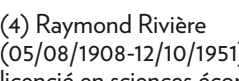

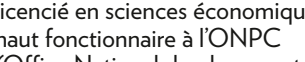
(Office National du placement

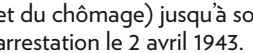

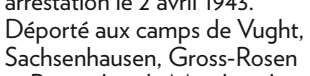
Sachsenhausen, Gross-Rosen
et Ravensbruck. Membre du

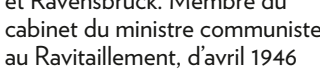
àvil 1947. Voir notice biographique par José Gotovitt
sur Maitron en ligne, hhtp:l/I

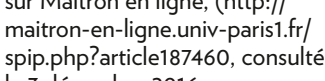
dembre 2016

(5) Citons Alphonse Roolandts
(vice-president): Rene Bodart (vice-president, Vincent Giets
(secretair genéral); Paule

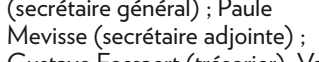

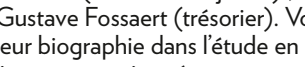
gre susmentiontesch

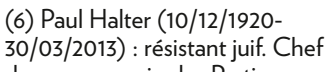
de corps au sein des Partisans

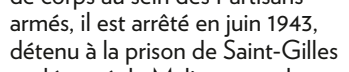
et déporté de Malines avec le le
transport du 20 septembre 1943. À Auschwitz, il sest affecté au

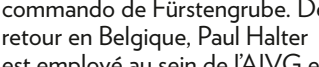
est employe au sein de liAIVG

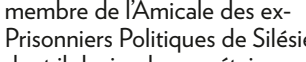

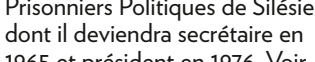
1965 et resididenten 1976. Voir
Dossier biographique Fondation (7) Dossiers SPF Securtite sociale,
Direction genenarale victimes dela

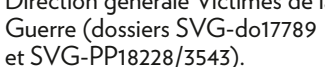
(8) Claire Duysburgh (o8/01/1/189-23/1/1/1/1800) resistante dans les reseaux
Comete ex Luc Marc. Aretée

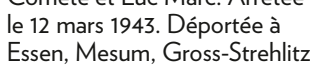
Essen, Mesum, Gross-strehlitz.
Devienta a son retour des camps responsable au ministere
a Reconstruction Service
a des Victimes de la Guerre),
vice-presidente de la CNPPA dont elle preside la section

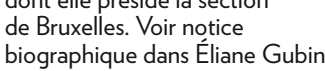

aux inciviques, défendre les droits des ex-prisonniers politiques et de leur famille et leur apporter un soutien moral et matériel.

L’assemblée élit Raymond Rivière 4 , militant communiste et rescapé du camp de Gross-Rosen, à la présidence de lassociation. Un comité constitué d’anciens déportés des camps de Gross-Rosen et Gross-Strehlitz, dont la plupart sont issus des rangs du Front de l'Indépendance et des Partisans armés, est également désigné5. L’Amicale de Silésie ne fait pas figure d'exception dans le paysage des associations de rescapés de laprès-guerre puisque la mouvance de gauche et en particulier le Parti communiste y sont fortement représentés. Par ailleurs, a linstar des autres amicales de camps, lAmicale de Silésie est a son origine exclusivement un groupement d anciens prisonniers politiques, les déportés juifs qui en sont membres le sont en raison de leur qualité de résistant. Il n'existe pas alors d'association spécifique pour la défense des droits des victimes de la déportation raciale qui, peu nombreuses, isolées et démunies, doivent prioritairement se reconstruire et se réintégrer dans la société belge. Ce sont des organismes d'entraide et de sociabilité issus de la communauté juive tels que Solidarité Juive ou l'AIVG (Aide aux Israélites Victimes de la Guerre) qui vont aider à leur réinsertion et jouer un rôle social, économique et culturel important.

Cependant, dans l'optique de réunir tous les survivants des camps selon le seul «critère de la souffrance», la CNPPA (Confédération nationale des Prisonniers politiques et ayants droit) est créée le 6 avril 1946, sous l'égide du ministre des Victimes de la Guerre. Le lendemain du congrès donnant naissance à la CNPPA, l'Amicale de Silésie, qui en est bien entendu membre, participe au grand rassemblement a de vil qui a lieu sur la Grand-Place de Bruxelles. Les differentes organisations La victimes du nazisme et les amicales de rescapés des camps sont ainsi unifiées. rassemblant résistants et déportés raciaux sur le même critère de souffrance, mais le projet rencontre l'opposition des catholiques et des associations patriotiques qui le rejettent, estimant que le critère déterminant delaqualité de prisonnier politique doitêtre exclitique Jice d'une prise de position officielle de l'Aricale de Silésie sur cette question, mais d'aprè les temoignages, elle a donné lieu à de vifs débats, certains étant contre le statu unique tandis que d'autres estimaient que les survivants devaient être au moin. bénéficiaires du statut de prisonnier politique. C'est à cette dernière demi-mesure qu’aboutit finalement la loi votée le 16 mars 1947, sorte de compromis «à la belge» par lequel tous les bénéficiaires ont les mêmes droits matériels, mais le titre de prisonnier politique est réservé aux seuls résistants. Les victimes juives sont donc exclues de toute reconnaissance officielle et ne peuvent bénéficier d'une mesure automatique de la part du ministère des Victimes de la Guerre que si elles peuvent prouver qu'lles étaient dans lásistance ou si elles sont de nationalité belge. Or $95 \%$ des Jufs déportés p. $210-215)$. Les antorites belge nécessité de mesures spécifiques à l'égard des rescapés juifs. L’opinion publique également ne s'intéresse guère aux victimes de la Solution finale et s'attache davantage, tout comme la presse, au destin glorieux des résistants. Le Fort de Breendonk acquiert un statut de symbole et cristallise toute l'attention, y compris celle de l'Amicale de Silésie qui prend part au pèlerinage annuel qui y est organisé. Pour Paul Halter ${ }^{6}$, futur président de l'Amicale, « On trouvait à ce moment-là que Breendonk suffisait amplement pour représenter la déportation et la résistance en Belgique. On n'avait pas tellement besoin finalement de mettre en exergue Auschwitz et ce que cela représentait rée cela représente symbolique, nebenéfie d'acuse renale elle retrouve son usage militaire. Au lendenain de la greste, la ménoire est donc essentiellement patriotique tandis que les persécutions antijuives disparaissent de la mémoire collective pour des décennies.

L’Amicale de Silésie quant à elle perd accidentellement son premier président en octobre 1951. A Raymond Rivière succède Adolphe D'Hont', ingénieur, ancien du Service de renseignements Zéro, également rescapé du camp de Gross-Rosen, mais contrairement à son prédécesseur il n'est pas membre du Parti communiste.

Parallèlement, Claire Duysburgh ${ }^{8}$ et Mariette Altorfer ${ }^{9}$ - qui deviendront des figures importantes de l'Amicale durant plus de vingt ans - sont nommées viceprésidentes.

En avril 1955, à l'invitation d'une association de résistants polonais, l'Amicale de Silésie emmène la délégation belge qui se rend à Auschwitz pour participer aux commémorations du $\mathrm{X}^{\mathrm{e}}$ anniversaire de la libération du camp. Une cérémonie, regroupant plusieurs milliers de personnes, représentant dix-sept pays, est organisée. Les participants visitent le camp, déposent une gerbe de fleurs et chaque délégation nationale est amenée à prononcer un discours. Celui de la délégation belge est représentatif de l'époque, plaçant les victimes de la Shoah dans l'ombre des héros de la Réristance loctocultantlas Résistance et occul responsable de l'Amicale, présent à la cérémonie: «Ce phénomène d'occultation ne nous a pas touchés. Pour les prisonniers juifs rescapés [...] revenant pour la première fois à Auschwitz, l'idéal de solidarité internationale était bien plus important pour l'avenir del'Europe et du monde que le rappel que les victimes les plus nombreuses des camps d'extermination nazis étaient juives.» (Goldstein, 2016, p. 203)

Claire Duysburgh va elle aussi dans le sens de l'occultation lorsqu'elle écrit quelques mois plus tard dans le Livre d’or du Musée d’Auschwitz: «C'est avec émotion que les Belges se sont recueillis sur les lieux où leurs compagnons de souffrance de dix-huitnations ont souffertetsontmorts pour avoir choisila libertép ${ }^{2}$ " Aujourd" symbole de la déportation et de l'exter n'est alors qu’une étape du premier voyage organisé par l'Amicale de Silésie en septembre 1955. Celui-ci a pour objectif principal un «pèlerinage à Gross-Rosen », camp
Dictionnaire des femmes belges
XIXe et $X X^{\circ}$ siecles, Bruxelles,
Racine 2006. . 229 .

(9) Mariette Génard êpouse d'Émile Altorfer. Militante communiste, membre du Front
de li lindépendance et de l'Armée belge des partisans. Arrêtée par
la Gestapo le 7 mai 1943 , elle est transtérée à la prison d'Essen à Papenburg, Kreuzburg et Jauer en Silésie. Après la guerre, elle reprend ses fonctions
dinstitutrice et sinvestit dans |Amicale des ex-Prisonniers Politiques de Silésie. Voir
notice biographique dans José Résistance et partic communiste, (10) « Parmi les dizaines de milliers de Belges que l'hitléris áráchés à eur pays, 27000 on
été amenés à Auschivitz. 1200 seulement, soit moins de $5 \%$ En ce même jour chez nous, été íncarcérés ete exécutétes les
résistants les milleurs fils de notre patrie, les prisonniers politiques de toute la Belgique
communient en pensées avec tous ceux qui ont subb loppression hitlerienne., Extrait de la déclaration de la délégation
belge - CarCob, Fonds Henri Buch, Farde 37.

(11) Maurice Goldstein :
(27/01/1922-06/10/1996) arrété le 3 septembre 1943 avec rafle de Bruxelles. Déportée par le $20^{\circ}$ convoi de Malines vers Auschwitz où il est affecté au
commando de Fürstengrube avant doccuper la fonction
dinfirmier à Auschitz. A son retour des camps, il intame des études de médecine. Il devient

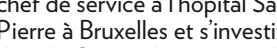
dans le Comité Internation
d'Auschwitz dont il devient president en 1977

(12) Daté du 12.09.1955 - Livre dor conservé au service des 
de concentration situé à environ 60 kilomètres de Wrocław (l'ancienne Breslau), dans l'ouest de la Pologne actuelle. Après avoir visité Gross-Rosen, les participants vingt-cinq personnes, des rescapés accompagnés de membres de leurs familles - se rendent àŚwidnica pour se recueillir devantle Monument aux prisonniers politiques, puis à Cracovie, Auschwitz, Nowa-Huta et sa «Fonderie Lénine» et terminent leur voyage par la visite de Varsovie.

S'il s'agit bien évidemment d'abord d'un «pèlerinage» en mémoire des disparus, ce voyage organisé par l'Amicale de Silésie en Pologne est empreint, comme en atteste le long compte-rendu qu'en fait Claire Duysburgh ${ }^{13}$, d'évidentes sympathies à l'égard du régime communiste polonais. À l'image de ce qui se passe dans le milieu des anciens déportés où la présence communiste crée d'importantes tensions, au sein de l'Amicale de Silésie, celles-ci aboutissent même au départ du président qui, selon de l'Amicale de Silésie, celles-ci aboutissent même au départ du président qui, selon Mariette Altorfer : «Ne voulait plus prendre la parole dans une amicale qu'il estimait être une amicale qui politiquement n'était pas soutenable.» Mariette Altorfer succède donc à Adolphe D’Hont au milieu de l’année 1956. Renée Van Hasselt'14 est désignée secrétaire nationale tandis que Claire Duysburgh complète ce trio féminin à la tête de l’organisation en gardant sa fonction de vice-présidente.

\section{LA PRÉSIDENCE DE MARIETTE ALTORFER ET LE MESSAGE ANTIFASCISTE} DE LAMICALE DE SILÉSIE (1956-1976)

Sous la présidence de Mariette Altorfer, six voyages - ou plus exactement «pèlerinages» - sont organisés vers les camps de Silésie. Le nombre de participants est relativement faible, à peine une quinzaine de personnes, pour l'essentiel des rescapés et leurs proches. Les voyages comportent la visite des camps de Gross-Rosen, GrossStrehlitz, parfois Jauer et Auschwitz et sont agrémentés de la visite de lieux touristiques comme Cracovie et Varsovie. Une cérémonie de dépôt de fleurs accompagnée

\section{du drapeau del'Amicale conclut toujours la visite d'un site (Grégoire, 1992 p. 13-17).}

Les prisonniers politiques ayant obtenu leur statut officiel et une reconnaissance nationale, une part importante de l'action de l'Amicale est consacrée aux activité commémoratives: anniversaires de la libération des camps, pèlerinages à Breendonk, ravivage de la flamme au Soldat inconnu, participation aux cérémonies d'autres amicales, inauguration de plaques et monuments, etc. L'Amicale, porte-drapeau en tête, est de (presque) toutes les cérémonies ! Parallèlement à ses «obligations » commémoratives, elle organise des événements, tels que l'organisation de débats et de projections de films, des représentations théâtrales, des concerts, dans le but de recueillir des fonds pour assurer son fonctionnement et pour alimenter ses œuvres sociales.

L’Amicale intervient également contre la libération d'anciens collaborateurs et apporte sa contribution dans la poursuite des criminels nazis. En 1947 déjà, elle était présente au procès de Rudolf Hoess, premier commandant du camp d'Auschwitz, qui comparaissait devant le tribunal suprême de Pologne du 11 mars au 2 avril. Des dizaines de témoins, anciens détenus de diverses nationalités étaient appelés à témoigner. Rayétaient appè́s à témoigner. Raymond Rivière encadrait alors la délégation belge en tant que prédident de linicale de Silesie. Une décennie plus tard, l'Amicale sert d'intermédiaire entre les victimes résidant en Belgique et la justice ouest-allemande. Lors de l'instruction du procès de Carl Clauberg,

médecin nazi d'Auschwitz, l'Amicale retrouve, à la demande du parquet allemand, d'anciennes détenues du bloc 10 d'Auschwitz, bloc des expérimentations médicales. Malheureusement, Carl Clauberg ne répondra pas de ses crimes puisqưil meurt en août 1957, quelques semaines avant de comparaître. L’Amicale de Silésie se charge également de trouver des témoins en Belgique dans le cadre du procès de Francfort, appelé également «second procès d’Auschwitz». Le procès qui se déroule du fort, appelé également «second procès d'Auschwitz». Le procès qui se déroule du
20 décembre 1963 au 19 août 1965, est public et permet de mettre en lumière les rouages de la vie concentrationnaire, mais surtout ceux de l'extermination. Dans la suite du procès Eichmann (1961), ce second procès d'Auschwitz, permet au grand public de prendre progressivement la mesure de l'ampleur du génocide des Juifs. En Belgique en revanche, si les premières études sur les persécutions antijuives datent également des années 1960, le pays mettra du temps à prendre ce tournant, les oppositions politiques etcommunautaires continuant à prere la guerre (Benvindo et Peeters, 2012, p. 231)

Parmi les autres missions de l'Amicale de Silésie figure la défense des intérêts de ses membres, notamment autour de problèmes administratifs auxquels ils sont
confrontés. L'aide de l'Amicale consiste en particulier à diffuser des renseignements confrontés. L'aide de l'Amicale consiste en particulier à diffuser des renseignements
sur les possibilités d'obtenir de l'Allemagne des indemnités auxquels ses membres
ont droit. En 1957, lorsque la société IG Farben est contrainte de verser des réparaont droit. En 1957, lorsque la société IG Farben est contrainte de verser des réparations aux anciens détenus du camp de Monowitz, commando de travail dépendant d'Auschwitz, l'Amicale de Silésie les oriente dans la procédure à suivre pour obtenir des dédommagements. C'est le cas encore en décembre 1959 lorsqu’un accord est conclu entre la Jewish Claims Conference ${ }^{15}$ et la firme Krupp au sujet des indemnisations. Les anciens détenus d'Auschwitz, de Gross-Rosen et des camps dépendants qui désirent déposer une demande d'indemnisation peuvent le faire par l'interméquiaire de l'Amicale.

\section{(15) La Conference on Jewish Germany, égalementa appelée Claims Conference ou Jewis
Claims Conference est une . dont le but est de réclamer des victimes du national-socialisme et les rescapés de la Shoah, mais

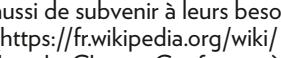

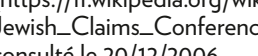


En Belgique, la législation allemande surl'indemnisation des victimesjuives està l'origine de la création d'une nouvelle association de déportés, l'Union des Déporté juifs et Ayants droit en Belgique, constituée au mois de février 1956. L’apparition de cette association, pour partie concurrente de l'Amicale de Silésie pose question et apparait comme un élément de scission important. Un certain nombre de rescapés juifs qui avaient le sentiment de ne pas être suffisamment pris en considération par l'Amicale de Silésie ont préféré rejoindre une organisation chargée de défendre spécifiquement leurs intérêts. Lorsqu'ils sont interrogés sur cette question trentecinq ans plus tard, les dirigeants de l'Amicale de Silésie déplorent cette scission. Ils réfutent l'idée d'une amicale qui n’aurait pas défendu les déportés juifs tout en admettant que, sur le plan belge: «Sil'Amicale de Silésie ne faisait pas assez pour le rescapés juifs, c'est en raison du cadre juridique qui existait [...] on ne trouvait pas les moyens juridico-techniques pour les défendre. ${ }^{16} \mathrm{Ils}$ rappellent que contrairement aux autres amicales de déportés politiques, l'Amicale de Silésie accueillait les déportés juifs qui n’étaient pas résistants. Tout porte à croire en effet que l'Amicale de Silésie a accepté assez vite l'adhésion de ceux-ci. La présence de Jacques Goldstein $^{17}$, non reconnu comme prisonnier politique et qui occupait même la fonction de trésorier de l'Amicale en 1955, ou celle de Maurice Goldstein au voyage de 1955 semblent confirmer cette hypothèse.

La création de cette association parallèle contraint néanmoins l'Amicale de Silésie à s'ouvrir plus formellement à l'ensemble des rescapés juifs, la majorité de ses membres demeurant des prisonniers politiques. La présidence de Mariette Altorfer va dans ce sens : «J'ai voulu changer la politique de l’Amicale. Au début, c'était les déportés avec leurs croix sur la poitrine, très fiers d'eux, d'en Mémorial aux martyrs juif

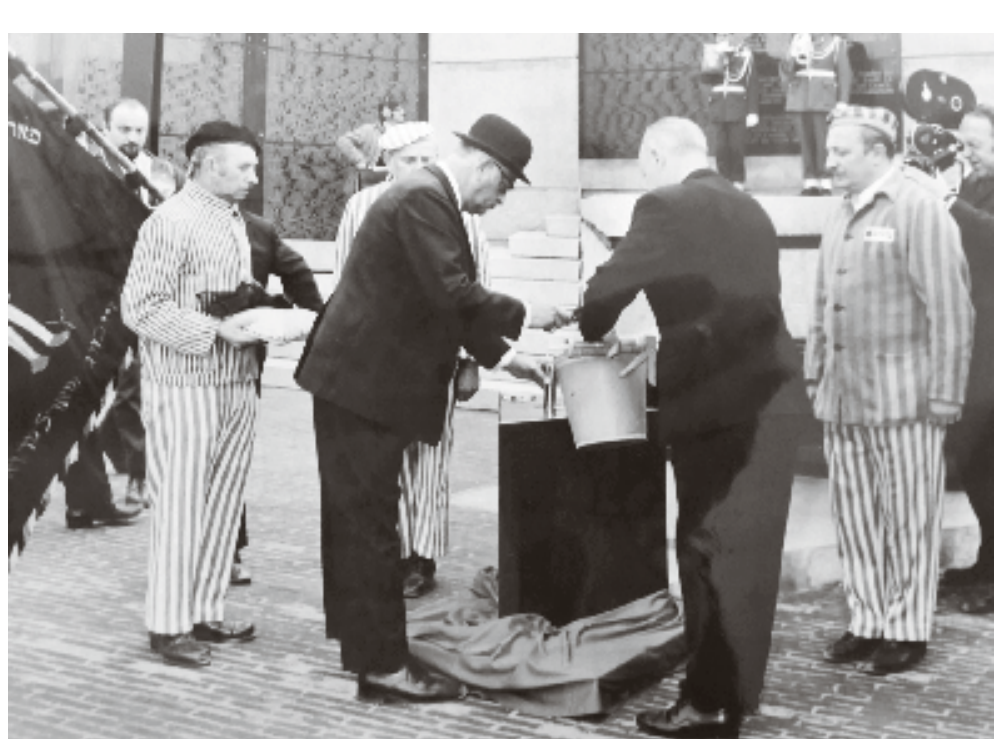
être sortis et qui ont derrière eux un passé de résistance. Et puis tu as toute la masse de ces gens qui eux n’ont pas tous un passé de résistant ct qui demandent à enterésistan et qui demandent à entrer dans la grande communauté de déportés.» Ceci amène l’Amicale de Silésie à se démarquer des autres amicales de déportés politiques et à adopter une posture «mixte» politique et juive Elle se met dès lors en porte à faux avec les autres associations de prisonniers politiques qui tiennent à maintenir avec force la distinction entre les différentes catégories de victimes. Aussi lorsque l'Union des Déportés juifs sollicite son adhésion uu sein de la Fraternelle des Amicales de Camps, celle-ci leur est refusée par la Fraternelle. Mariette Altorfer explique avoir tenté en vain de la convaincre : «La Fraternelle a mis en avant le fait qu'ils n'étaient que les bénéficiaires du statut et qu'ils n’étaient pas des prisonniers politiques consacrés. J’ai demandé qưon fasse attention et j’ai soulevé le critère de la souffrance en disant que c'était la base sur laquelle il faudrait juger, mais je n’ai pas remporté le morceau. Je n’ai peut-être pas été assez convaincante.» Quoi qu'il en soit - et même s'il est impossible de déterminer dans quelle mesure il y a transfert de memos l'Amicale de Silésie vers l'Union des 政 micale de Silésie à ce moment-là, d'autres sont membres des deux associations.

La création de l'Union des Déportés juifs de Belgique provoque une accélération mmédiate de la commémoration de la persécution juive en Belgique. Elle organise 列 lérection du Mémorial inauguréle 19 avril 1970 à Anderlecht. Avec ce monument, les victimes juives entrent tardivement en concurrence avec les prisonniers politiques qui depuis plus de vingt ans se réunissaient à Breendonk. De son côté, l'Amicale de Silésie tient un discours universaliste qui ne nomme pas la persécution juive par son nom, on y parle de victimes, pas de victimes juives. L'Amicale de Silésic s'insson nom, on y parle de crit dans la tradition antifasciste qui était d'additionner les victimes qui mouraient d'épuisement et de mauvais traitements dans les camps de concentration à celles qui étaient destinées à une disparition immédiate dans les centres de mises à mort. Cette tradition antifasciste dans laquelle s'inscrit l'Amicale de Silésie intègre aussi le pacifisme. Antifascisme et pacifisme vont de pair dans ces années 1950-1960, années de Guerre froide qui mettent les questions liées à la paix et à la prolifération des armes atomiques au centre des préoccupations de l'Amicale. Le combat antifasciste d'avant-guerre se poursuit donc dans la lutte pour la paix, et le discours pacifiste de l'Amicale de Silésie entend puiser dans l'expérience des camps. Dans ce climat de Guerre froide, l'Amicale, par sa composition - nombre de ses membres ont des sympathies communistes prononcés -, adopte une idéologie générale anti-atlansympathies con tiste en se positionnant contre le rearmement de la RFA et l'implantation des bases américaines en Belgique. L'Amicale de Silésie adhère à l'UBDP - l'Union belge pour la défense de la paix (UBDP), organisation cryptocommuniste ${ }^{18}$ et au Mouvement du 8 mai $^{19}$, mouvement liéà la perception du péril atomique par l'opinion publique. L'Amicale prend ainsi part à de nombreuses manifestations pour la paix et contre la prolifération des armes atomiques : participation aux marches antiatomiques et autres «circuits pour la paix», conférences et actions en faveur de la détente internationale.

L'orientation antifasciste de l'Amicale de Silésie apparaîtégalement dans la première exposition qu'elle conçoit pour le «pavillon belge» du Musée d'Auschwitz. En 1957, le Comité international d'Auschwitz (fondé en 1952 et composé des membres des différentes organisations nationales) décide de l'internationalisation
(18) Créée en 1949 par le PCB avec la collaboration de membres branche belge du Conseil mondial de la Paix, IUBDP sinvestit dans une série d'actions liées spécifiquement à la situation
nationale telle lopposition aux bases militaires americaines en Belgique, la politique colonial durée du service militaire. (19) «Les mouvements de la paix en Belgique », in Courrier
hebdomadaire du CRISP, 1964/15. 
du site et propose que chaque pays qui en fait la demande puisse obtenir une salle d'exposition dans l'un des blocs d'Auschwitz. En Belgique, c'est l'Amicale de Silésie qui prend l'initiative de la réalisation d'un pavillon national (Thanassekos, 2008 , p. 177-184). Elle est chargée d'élaborer le scénario de l'exposition, un scénario «qu reflète le visage de la Résistance en Belgique et du lourd tribut qu’elle a payé pour recouvrer sa liberté. $»^{20}$ Bien que la déportation juive ne soit pas occultée dans le scénario de l'exposition, elle apparaît néanmoins en dernier lieu et ne représente qu'un cinquième de l'exposition. Elle est intégrée dans l'ensemb de la criminatié extinalí nazie con comme un tout. Le pavillon belge ne filt pas excent les expositions presentees dans les pavillons nationaux sont avant tout axees sur l'histoire de chacun des États durant la guerre, souvent sans rapport avec Auschwitz et les principales victimes du camp (Brutmann, 2015, p. 101). Sa réalisation et surtout son déménagement en Pologne prendront du temps, si bien que l'exposition belge ne sera inaugurée qu’en 1965.

Au mois d'avril 1967, deux ans après l'inauguration du pavillon belge, une importante délégation de l'Amicale de Silésie se rend à Auschwitz pour l'inauguration du Monument international, nouveau lieu de commémoration pour les cérémonies officielles. En Belgique, l'Amicale de Silésie sert de relais au projetet se charge tou Durge tout d'abord de constiture une activité intense pour récolter des fonds pour fint tions auprès des autorités, collectes, galas, mais surtout l'organisation d'activité culturelles dont la plus prestigieuse d'entre elles, la venue au Palais des Beaux-Arts de Bruxelles de David Oïstrakh, un des violonistes les plus réputés du XXe siècle.

\section{LAMICALE DE SILÉSIE DEVIENT AMICALE D'AUSCHWITZ (1976)}

À la fin des années 1970, l’Amicale de Silésie s'essouffle. Elle remplit son cahier de charges sur le plan des commémorations, mais peine à se renouveler et à se redéployer. Les activités de l'as déportés età leurs rant»conmeledép l'Amicale demande à être déchargée de ses fonctions. Une réflexion est alors lancée auprès des membres en mars 1976. Une circulaire propose de nouvelles orientation. sur lesquelles les membres de l'Amicale sont amenés à se prononcer. Les retours sont positifs. Un nouveau comité exécutif restreint est alors constitué, emmené pa Paul Halter, secrétaire de l'Amicale depuis 1965. Celui-civa relancer des actions non plus simplement de commémoration, mais axées vers la transmission et la conservation de la mémoire du phénomène concentrationnaire. L’assemblée générale du 13 juin 1976 entérine la démission de l'ancien comité Mariette Altorfer et Claire Duysburgh deviennent présidentes d'honneur de l'Amicale. Paul Halter prend la . général. Maurice Goldstein, qui devient par ailleurs président en 1977 du Comité

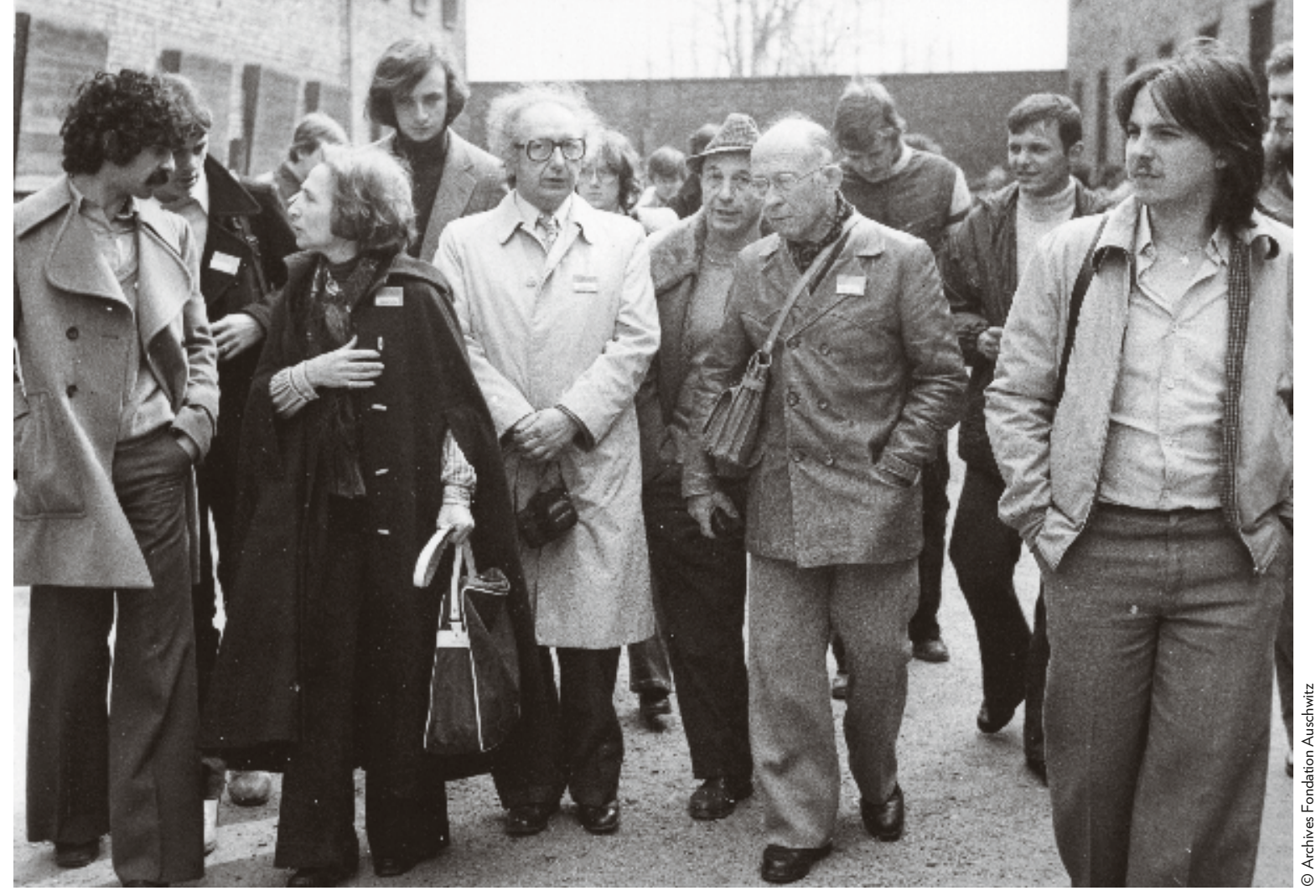

- Voyage des jeunes à

international d'Auschwitz et René Raindorf jouent également un rôle important dans cette nouvelle direction.

Afin d'être plus efficace dans la lutte contre le négationnisme, l'Amicale de Silésie change de nom et devient en octobre 1976, l'Amicale Belge des ex-Prisonniers Politiques d'Auschwitz-Birkenau, camps et prisons de Silésie ou souvent nommée Amicale Belge d'Auschwitz. Le terme de «Silésie» passe au second plan au profit d’Auschwitz : «Nous avons estimé devant le phénomène des négationnistes qu'il fallait changer même le nom de l’Amicale parce que ce n’était pas assez clair, qu'il fallat se battre sur Auschwitz beaucoup plus que nous le croyions p (1) et l'Amicale de Silésie est devenue Amicale d'Auschwitz „(René Raindorf)

Ce changement de nom intervient à un moment où Auschwitz devient dans la conscience collective le symbole de la déportation. Les membres de l'Amicale se présentent également de plus en plus comme des déportés raciaux. Les valeurs sur lesquelles s'est construite la mémoire de la Résistance s'effritent, la résistance communiste en particulier est effacée des mémoires.

Les voyages en Pologne organisés par l'Amicale témoignent eux aussi de cette évolution et s'inscrivent dans la lutte contre le négationnisme. Ils se déroulen désormais uniquement à destination du seul complexe d’Auschwitz-Birkenau et abandonnent leur car giques etvecteurs de la 
l'ensemble des activités de l'Amicale pendant un an et demi, est organisé en 1978 et emmène cent vingt jeunes universitaires à Auschwitz. Ils sont accompagnés de dix rescapés qui ont pour mission de leur transmettre leur expérience concentrationnaire sur les lieux mêmes de leur détention. Mais pour le comité, le bilan du voyage est à nuancer. Si les étudiants sont passionnés par les récits des rescapés et lient avec eux des liens fraternels, d'un point de vue de l'acquis et surtout de la transmission de l'expérience, le comité considère qu'il manque aux étudiants les prérequis nécessaires pour en tirer des conclusions générales.

Le voyage suivant, organisé en 1980, est dès lors destiné aux enseignants, ceuxci apparaissant plus comme des relais. Comme pour le voyage précédent, l’Amicale d’Auschwitz déploie une activité intense pour trouver des fonds en s'adressant notamment aux mandataires politiques dont le soutien est essentiel. Le voyage encadre une soixantaine de personnes et s'étend sur une petite semaine avec la visite de Varsovie et de Cracovie. La formule du voyage des professeurs est considérée comme une réussite et sera rééditée annuellement à partir de 1982 par l'Amicale d'Auschwitz ou plus exactement par la Fondation Auschwitz qui lui succède.

\section{CONCLUSION : UNE FONDATION POUR PRENDRE LE RELAIS.}

C'est en juillet 1980 que la Fondation Auschwitz voit le jour, se substituant progressivement à l'Amicale Belge d'Auschwitz, dont elle poursuivra les activités dans le sens de la préservation et de la transmission de la mémoire de la déportation. Le problème de la disparition des témoins a préoccupé l'Amicale d'Auschwitz dès la fin des années 1970. Cette crainte, parallèlement à la volonté de lutter contre le négationnisme, a amené l'Amicale d'Auschwitz à vouloir pérenniser son action à travers un nouvel instrument: «Voyant les témoins des camps disparaître chaque année, l’Amicale décide de créer une nouvelle association qui permettra à la nouvelle

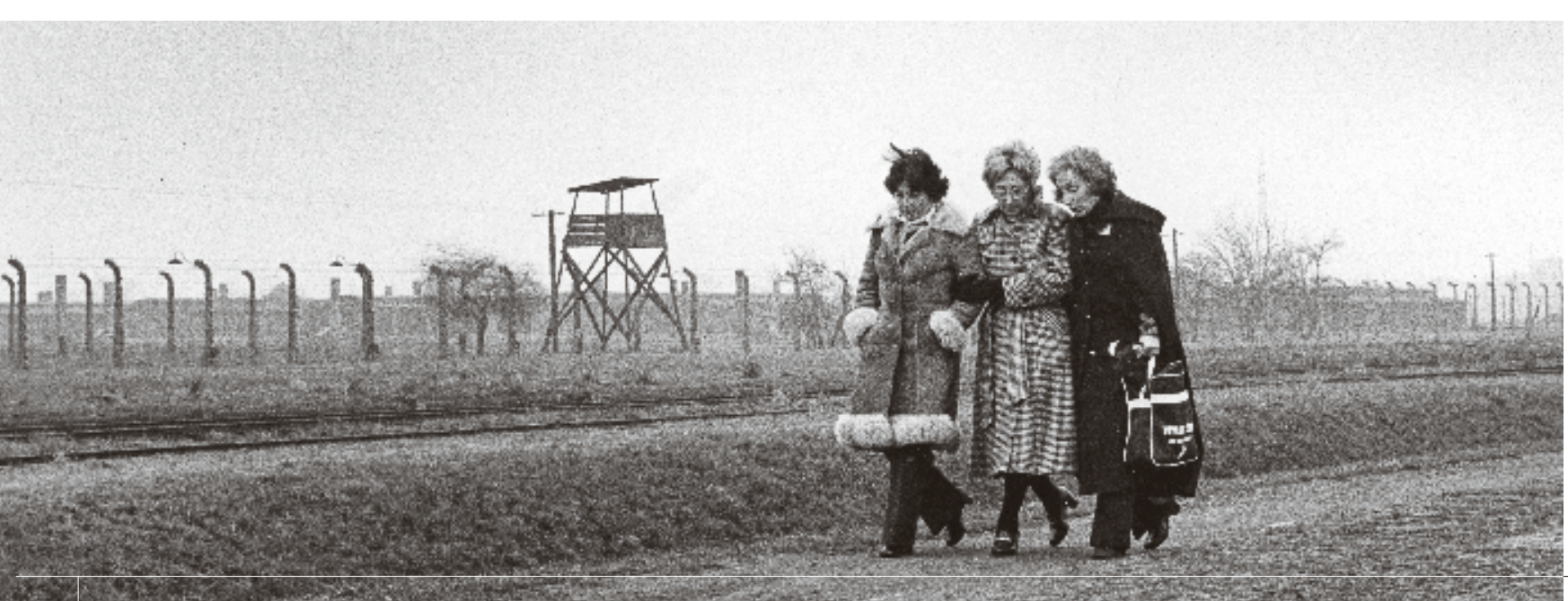

génération de pouvoir participer à ses actions et de les développer. Dans l'esprit de ses fondateurs, la Fondation Auschwitz doit prendre la relève de l'Amicale dans les années à venir. $»^{21}$

L'histoire de cette Fondation ne fait pas l'objet du présent article, mentionnons néanmoins qu'elle se dotera rapidement d'un centre de documentation et d'une bibliothèque, s'occupera de la conception du deuxième pavillon belge à Auschwitz penregistrements audio et ensuite audiovisuels de témoignages de rescapés entre autres.

La Fondation Auschwitz est créée à un moment où en Belgique, l'intérêt pour les victimes juives du nazisme connaît un essor considérable. Cet intérêt pour la persécution des Juifs s'explique en partie par les actions menées en 1980 contre es responsables allemands de la déportation en Belgique dans le cadre du procès de Kiel. Ce procès bénéficie d'une large attention médiatique en Belgique, tant au sein de la communauté juive qu'en dehors et un public croissant prend conscience de l'ampleur de l'extermination des Juifs de Belgique. Parallèlement, la communauté juive commence à réinterroger son propre passé. (Benvindo et Peeters, 2012 p. 234). De nombreux témoins sont à présent prêts à transmettre leur expérience p. 234). De no lour experience de l'Occupation aux jeunes generations. La communaute juive prend conscience de l'importance mémorielle de la caserne Dossin qui deviendra en 1995 le Musée juif de la Déportation et de la Résistance. De leur côté, les historiens investissent progressivement ce domaine de recherche. Maxime Steinberg qui avait posé les premiers jalons de l'étude du judéocide en Belgique publie au milieu des années 1980 sa trilogie L'étoile et le fusil, considérée comme l’œuvre de base sur ce sujet. Il met notamment en lumière la participation de certaines autorités publiques belges dans l'accomplissement de la politique de persécution des Juifs sous l'Occupation.

La montée de l'extrême droite dans les années 1990 et le poids croissant du genocide dans la conscience collective amènent à considérer la persén Juifs comme un événement unique. La classe politique manifeste un intérêt croissant pour la Seconde Guerre mondiale et intervient dans le champ mémoriel, ce qui a pour conséquences de bouleverser les rapports de force entre les différentes mémoires. Breendonk est transformé en Human Rights Memorial à l’occasion de la célébration du cinquantième anniversaire de la fin de la guerre tandis quau MuséeMémorial Kazerne Dossin, la question des Droits de l'homme est au centre des préoccupations des autorités flamandes, responsables du musée. «Une nouvelle conception de l'éducation à la citoyenneté triomphe, dans laquelle le conflit 19401944 fait fonction d'avertissement moral et politique.» (Benvindo, 2012, p. 69) Plus

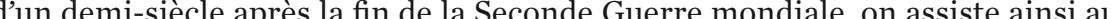
par d'une mém de l'homme dans laquelle le genocide des Juifs est central, laissant les mémoires patriotiques et antifascistes au passé.

\section{(21) Rapport d'activité de la}

BIBLIOGRAPHIE

- Benvindo, Bruno (2012):

«Autorités du passé. Mémoires

Breendonk $1944-2010$ ", Revie
Brend

XLII, 2-3,3. p. 48-77.

- Benvindo, Bruno et Peeters,

dela la gurre: Les décombres

en conflit 1945-2010, Waterloo

Brutmann, Tal (2015)

Goldstein, Maurice (2016)

Chroniques d'un rescape

belge né en Pologne, Bruxelles,

Grégoire, Jocelyn (1992):

Rapport sur le fonds d'archives
de l'Amicale des ex Prisoniers

Politiques $d$ 'Auschwitz-Birkenau

Fondation Ausch de Silésie,

non publié. Disponible en

ligne : $h$ ttp: ://www.gregoire-
tinant.be/amicalesilesie-texte/

amicalesilesie.htm, consulté le

- Lagrou, Pieter (2003)

Mémoires patriotiques et
Occupation nazie. Resistants

quis et déportés en Europe

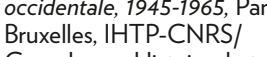

Complexe, «Histoire du temps

Thanassekos, Yannis (2008)

"Du premier au troisième
pavillon belge à Auschwitz

(1966-2006) $)$, in Béatrice
(1)

Pleury et Jacques Walter
(dir.), Qualifier des lieux de

(dir.), Qualifier des lieux de
détention et de massacres (2),

détention et de massacres (2),
Metz, Université Paul Verlaine.
p. 177-184 